\title{
Low-dose Microscopy and Beam Damage Study of Infiltrated Zeolite Y
}

\author{
Parivash Moradifar ${ }^{1}$, Yunzhi Liu ${ }^{2}$, Hiu Yan Cheng ${ }^{2}$, John Badding ${ }^{3}$, Nasim Alem ${ }^{1}$ \\ ${ }^{1}$ Department of Materials Science and Engineering, Materials Research Institute, The \\ Pennsylvania State University, University Park, USA \\ ${ }^{2}$ Department of Chemistry, The Pennsylvania State University, University Park, USA \\ ${ }^{3}$ Department of Physics, Department of Chemistry, Department of Materials Science and \\ Engineering, The Pennsylvania State University, University Park, USA
}

\begin{abstract}
Zeolites are aluminosilicate crystalline materials consist of $\mathrm{Si}(\mathrm{Al}) \mathrm{O}_{4}$ units, with aluminum atoms occupying some of the tetrahedral sites in silicon [1]. The interconnected array of aluminasilicate cages result in the formation of three-dimensional open channels within molecular size range of 0.3 to $1.5 \mathrm{~nm}$, thus providing high specific surface area in zeolite [2, 3]. This high surface area makes zeolite a promising candidate for a wide range of applications in catalysis, separation, ionexchange, and molecular-sieve sorbents $[4,5]$. Zeolites can also work as 3D ordered nanotemplates infiltrated with different atomic species to create metalattice materials exhibiting both quantum confinement and long-range transport. The infiltrated zeolites can have diverse electronic, optical and magnetic applications such as solar cells, near-IR photonics, light emitting devices, and improved thermoelectrics.
\end{abstract}

Structure determination of zeolites can be challenging by conventional X-ray diffraction methods due to the small crystallite size of zeolites [1]. In contrast, high-resolution scanning/transmission electron microscopy (HR-S/TEM) can provide direct observation of the complex zeolite frameworks and their atomic structure. In this study, we investigate the atomic structure of zeolite frameworks using ultra-high resolution aberration-corrected S/TEM imaging before and after infiltration. Zeolites are sensitive to electron beam irradiation and can undergo beam damage during imaging $[1,5,6]$. As a result, recent investigations have focused on the beam damage behavior of empty zeolites through low dose imaging [5-9]. Using low dose imaging technique, we image the structure of the zeolite frameworks and determine their resistance to beam damage before and after infiltration $[9,10]$.

This study will present the atomic and chemical structure that occurs in infiltrated zeolite Y (using metallic and semiconducting species such as $\mathrm{Pt}$ and $\mathrm{Ge}$ ) under electron beam irradiation. Figure 1a, shows a high resolution image of Pt infiltrated-zeolite at $80 \mathrm{kV}$. Figure $1 \mathrm{~b}$, shows the degradation of crystal and formation of amorphous area at the edge of the sample as a result of electron beam irradiation while the lattice spacing confirms no structural transformation. This study will also determine the critical dose [1] after infiltration of zeolite $\mathrm{Y}$ with metals or semiconductors. Imaging the atomic and chemical structure of zeolite frameworks and understanding the damage mechanism and structural evolution under the electron beam can provide information on how well the nano-pores in this structure are filled and how the infiltration of zeolite $\mathrm{Y}$ can enhance its resistance to electron beam irradiation. This understanding is the key to develop novel zeolite-based metalattice materials for future optical, magnetic and electronic applications. 
References:

[1] M. Pan and P. A. Crozier, Ultramicroscopy 48 (1993), P. 332.

[2] S. Mintova, N. H. Olson, and T. Bein, Angewandte Chemie International Edition 38 (1999), P. 3201.

[3] V. Ortalan, A. Uzun, B. C. Gates, and N. D. Browning, Nature nanotechnology 5 (2010), P. 506.

[4] S. B. Rice, J. Y. Koo, M. M. Disko, and M. M. J. Treacy, Ultramicroscopy 34 (1990), P. 108.

[5] M. M. J. Treacy and J. M. Newsam, Ultramicroscopy 23 (1987), P. 411.

[6] R. Csencsits and R. Gronsky, Ultramicroscopy 23 (1987), P. 421.

[7] Y. Yokota, H. Hashimoto, and T. Yamaguchi, Ultramicroscopy 54 (1994), P. 207.

[8] R. Csencsits and R. Gronsky, Zeolites 8 (1988), P. 122.

[9] O. Ugurlu, J. Haus, A. A. Gunawan, M. G. Thomas, S. Maheshwari, M. Tsapatsis, and K. A. Mkhoyan, Physical Review B 83 (2011), P. 113408.

[10] B. Barton, Microscopy and Microanalysis (2012).

This work was funded by the Penn State MRSEC, Center for Nanoscale Science, under the award NSF DMR-1420620.
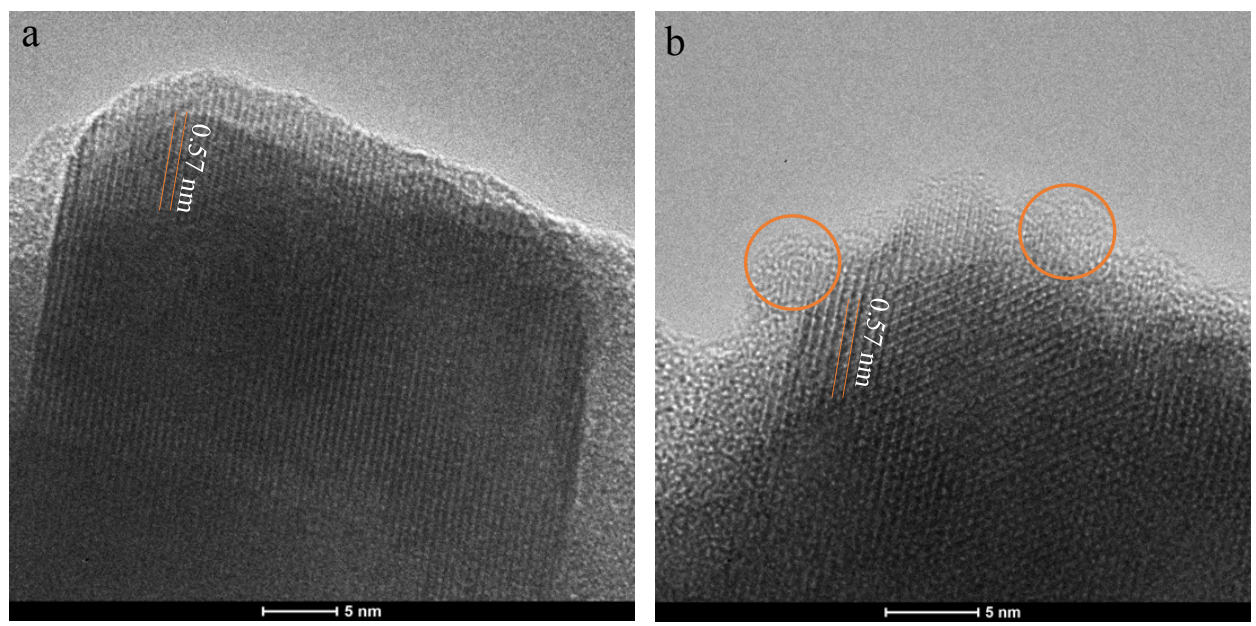

Figure 1. (a) HRTEM image of Pt infiltrated zeolite $\mathrm{Y}$ at $80 \mathrm{kV}$ (b) degradation of Pt infiltrated zeolite $\mathrm{Y}$ under electron beam at $80 \mathrm{kV}$ 